\title{
Output Feedback Control for Mobile Robot Systems with significant External Disturbances
}

\author{
Kang $\mathrm{Wu}^{1,2}$, Jinya $\mathrm{Su}^{2,1} \&$ Changyin $\mathrm{Sun}^{1^{*}}$ \\ ${ }^{1}$ School of Automation, Southeast University, Key Laboratory of Measurement \\ and Control of CSE,Ministry of Education, Nanjing 210096, China; \\ ${ }^{2}$ Department of Aeronautical and Automotive Engineering, \\ Loughborough University, Loughborough LE11 3TU, United Kingdom \\ Citation Wu K, Su J Y, Sun C Y. Output Feedback Control for Mobile Robot Systems with Non-vanishing \\ External Disturbances. Sci China Inf Sci, for review
}

\begin{abstract}
Dear Editor,
The control of nonholonomic systems, like those describing mobile robots, has attracted considerable research attention in recent decades due to its theoretical and practical importance. Brockett's theorem states that nonholonomic systems cannot be stabilized to an equilibrium point by any source of smooth or continuous state feedback. Additionally, the required accuracy of measuring the system states is often unachievable in practice. These factors introduce considerable difficulty to the problem of controlling mobile robot and other nonholonomic systems.
\end{abstract}

Several studies have addressed the output feedback stabilization of uncertain nonholonomic systems[1-3]. However, these studies require the first subsystem of the considered system to be linear and known, and the virtual control directions also need to be known. Some researchers have attempted apply output feedback control when the first subsystem has uncertain parameters. One example uses a constructive observer design to control a mobile robot model[1]. Recently, a novel adaptive output feedback controller for global stabilization of nonholonomic systems in chained form was provided for a system with unknown virtual control directions[4]. The effects of non-vanishing external disturbances were not con-

* Corresponding author (email: cysun@seu.edu.cn) sidered in the design of these controllers, although most practical systems are affected by significant external disturbances.

Several methods are available for solving the disturbance problem[5-7]. In general, to reject the external disturbances, the extended state observer (ESO) [5] is used in active disturbance rejection control. Additionally, the disturbance observerbased control technique provides a promising approach to handle the system disturbances[6]. For the mobile robot systems, good results for stabilization and tracking control have been reported when the nonholonomic constraints are precisely known[8]. However, the presence of external disturbances often introduces dynamic constraints because of parameter uncertainties. To the best of our knowledge, very few reports have proposed output feedback for use in mobile robot systems or other nonholonomic systems that require antiinterference control.

Motivated by the above observations, this letter proposes a robust output feedback controller for use in mobile robot systems that suffer parametric uncertainties, deviation in the measured angles, and non-vanishing external disturbances. The proposed controller is designed to handle two challenges in a nonholonomic system: the unknown system parameters and the presence of non- 
vanishing external disturbance. First, these challenges are overcome by generalizing the disturbance as an extended state. Second, an ESO is constructed with the gain from an off-line timevarying Riccati matrix differential equation to estimate the unmeasurable states. These two strategies yield a controller that applies time-varying output feedback. The proposed robust controller drives the system states to the origin asymptotically.

In the presence of parametric uncertainties and non-vanishing external disturbances, the dynamics of a unicycle-type mobile robot with angle measurement errors can be described as

$$
\begin{aligned}
& \dot{x}_{c}=p_{1}^{*} v \cos (\theta+\varepsilon)+c_{0} x_{c}, \\
& \dot{y}_{c}=p_{1}^{*} v \sin (\theta+\varepsilon), \\
& \dot{\theta}=p_{2}^{*} \omega+d(t)
\end{aligned}
$$

where $c_{0} x_{c}$ is the horizontal drift, $p_{1}^{*}$ and $p_{2}^{*}$ are bounded unknown positive parameters determined by the radius of the rear wheels, $d(t)$ is the timevarying external disturbance, and $\varepsilon$ is a small bias orientation. This letter assumes that $d(t)$ can be decomposed into $d(t)=d_{1}(t) d_{2}(t)$, where $d_{1}(t) \neq 0$ is known and bounded, and $d_{2}(t)$ is unknown but $\dot{d}_{2}(t) \in l_{2}$, i.e., $d(t)$ is a time-varying disturbance that is relatively fast. The control problem is steering the robot toward the origin while not knowing the values of unknown parameters, the angle measurement error, or the direction and magnitude of an external disturbance. Indeed, if the external interference, angle deviation, and nonlinear drift are ignored, and all state variables are measurable, the model (1) is a classical nonholonomic system. The second-order approximation of system (1) near $\theta=0$ is given by[1] as follows

$$
\begin{aligned}
& \dot{x}_{l}=p_{1}^{*}\left(1-\frac{\varepsilon^{2}}{2}\right) v+c_{0} x_{l}, \\
& \dot{y}_{l}=p_{1}^{*}\left(\theta_{l} v+\varepsilon v\right), \\
& \dot{\theta}_{l}=p_{2}^{*} \omega+d(t) .
\end{aligned}
$$

If the coordinates are transformed as follows, $x_{0}=x_{l}, x_{1}=y_{l}, x_{2}=\left(\theta_{l}+\varepsilon\right), u=\omega, u_{0}=v$, the system can be rewritten as

$$
\begin{aligned}
& \dot{x}_{0}=p_{1}^{*}\left(1-\frac{\varepsilon^{2}}{2}\right) u_{0}+c_{0} x_{0}, \\
& \dot{x}_{1}=p_{1}^{*} x_{2} u_{0}, \\
& \dot{x}_{2}=p_{2}^{*} u+d(t) .
\end{aligned}
$$

Evidently, this system is a third-order nonholonomic system in chained form, and if the perturbation $\varepsilon$ is unknown, the state $x_{2}$ is not measurable.
To handle the unknown parameters $p_{1}^{*}$ and $c_{0}$, we can take $u_{0}$ for the first subsystem of (3) as

$$
\dot{u}_{0}=-k_{1} \operatorname{sgn}\left(g_{0}\right) x_{0}-k_{2} u_{0},
$$

where $g_{0}=p_{1}^{*}\left(1-\varepsilon^{2} / 2\right)$ and the gains $k_{i}$ 's are assigned such that the equation

$$
s^{2}+\left(k_{2}-c_{0}\right) s+k_{1}\left|g_{0}\right|-k_{2} c_{0}=0,
$$

has two negative real roots. As $g_{0}$ and $c_{0}$ are bounded, values of $k_{1}$ and $k_{2}$ that satisfy equation (5) are determined easily (if $c_{0}$ is negative, we can simply choose $k_{1}=0$ and $k_{2}>0$, i.e., neither the sign of $g_{0}$ nor the bounds of $g_{0}$ and $c_{0}$ need to be known) The aforementioned design guarantees that $x_{0}$ and $u_{0}$ are convergent to zero exponentially and $\dot{u}_{0} / u_{0}$ is measurable and bounded for small perturbations $\varepsilon$.

To construct the Kalman filter observer for subsystem $x$, we introduce another transformation $\zeta_{1}=\left(1 / p_{1}^{*} p_{2}^{*}\right) x_{1}, \zeta_{2}=\left(1 / p_{2}^{*}\right) x_{2}$ and find that

$$
\begin{aligned}
& \dot{\zeta}_{1}=\zeta_{2} u_{0}, \\
& \dot{\zeta}_{2}=u+\left(1 / p_{2}^{*}\right) d(t) .
\end{aligned}
$$

Using the change of variables for a nonholonomic system $z_{1}=\zeta_{1} / u_{0}, z_{2}=\zeta_{2}$ and further letting $z_{3}=\bar{d}(t)=\left(1 / p_{2}^{*}\right) d_{2}(t)$, we have the following dynamics

$$
\begin{aligned}
& \dot{z}_{1}=-\frac{\dot{u}_{0}}{u_{0}} z_{1}+z_{2}, \\
& \dot{z}_{2}=u+d_{1}(t) z_{3}, \\
& \dot{z}_{3}=\dot{\bar{d}}(t)=h(t) .
\end{aligned}
$$

Notbly $z_{i}$ is not available in the design of controller because the parameters $p_{1}^{*}$ and $p_{2}^{*}$ are unknown. Conversely, $p_{1}^{*} p_{2}^{*} z_{1}$ is an available signal in the controller design. Let $z=\left[z_{1}, z_{2}, z_{3}\right]^{T}$, the dynamics can be rewritten into the following compact form

$$
\dot{z}=A(t) z+C_{2} u+C_{3} h(t),
$$

where $A=\left[-\dot{u}_{0} / u_{0}, 1,0 ; 0,0, d_{1}(t) ; 0,0,0\right], C_{2}=$ $[0,1,0]^{T}, C_{3}=[0,0,1]^{T}$. The extended state Kalman observer can be given as follows[9]

$$
\dot{\widehat{z}}=A(t) \widehat{z}+C_{2} u-P C_{1} C_{1}^{T} \widehat{z},
$$

where the observer gain $P(t)$ is updated by the following time-varying Riccati differential equation

$$
\left\{\begin{array}{l}
\dot{P}=P A^{T}(t)+A(t) P-P C_{1} C_{1}^{T} P+I, \\
P(0)=P_{0}>0, \quad C_{1}=[1,0,0]^{T} .
\end{array}\right.
$$

This Riccati differential equation is solvable. The observation error variables are defined as $e_{i}=$ $z_{i}-\widehat{z}_{i}, i=1,2,3$, so the error dynamics satisfies

$$
\dot{e}=\left(A(t)-P C_{1} C_{1}^{T}\right) e+P C_{1} z_{1}+C_{3} h(t) .
$$


Let $\sigma_{1}=\widehat{z}_{2}-\alpha_{1}$, we get

$$
\begin{aligned}
\dot{z}_{1}= & -\frac{\dot{u}_{0}}{u_{0}} z_{1}+z_{2} \\
& -\frac{\dot{u}_{0}}{u_{0}} z_{1}+e_{2}+\sigma_{1}+\alpha_{1}, \\
\dot{\widehat{z}}_{2}= & d_{1}(t) \widehat{z}_{3}-p_{21}(t) \widehat{z}_{2}+u, \\
\dot{\widehat{z}}_{3}= & -p_{31}(t) \widehat{z}_{3},
\end{aligned}
$$

where $p_{21}(t)$ and $p_{31}(t)$ are the corresponding elements of the matrix $P(t)$.

Although the term $z_{1}$ is unknown, the term $p_{1}^{*} p_{2}^{*} z_{1}$ is measurable. Applying the backstepping method to the equations (12)-(14), we determined that there exist proper (large) positive constants $K, L_{1}$ and $L_{2}$ such that the controller defined by

$$
\begin{aligned}
\alpha_{1}= & -K p_{1}^{*} p_{2}^{*} z_{1}, \\
u=- & d_{1}(t) \widehat{z}_{3}+p_{21}(t) \widehat{z}_{2}+K p_{1}^{*} p_{2}^{*} \frac{\dot{u}_{0}}{u_{0}} z_{1} \\
& -L_{1} \operatorname{sgn}\left(\sigma_{1}\right) K\left|p_{1}^{*} p_{2}^{*} z_{1}\right|-L_{2} \sigma_{1} .
\end{aligned}
$$

can drive the states $z_{1}, e, \sigma_{1}$ to zero and $z_{3}$ and $\widehat{z}_{3}$ are bounded. Furthermore, it follows that the states $\zeta_{1}, \zeta_{2}$ and $x_{1}, x_{2}$ converge to zero. This convergence also ensures that the $\left(x_{l}, y_{l}, \theta_{l}\right)$ converges to the equilibrium $(0,0,-\varepsilon)$.

Theorem 1. For system (2), if the non-vanishing disturbance $d(t)$ satisfies $d(t)=$ $d_{1}(t) d_{2}(t)$, where $d_{1}(t) \neq 0$ is known and bounded, $d_{2}(t)$ is unknown, but $\dot{d}_{2}(t) \in l_{2}$, the unknown parameters $p_{1}^{*}$ and $p_{2}^{*}$ are positive and bounded, and the controller is set as (15)-(16), then all signals in the closed-loop system are bounded and $\left(x_{l}, y_{l}, \theta_{l}\right)$ converges to the equilibrium $(0,0,-\varepsilon)$.

Numerical results. We assumer that $p_{i}^{*} \in$ $[0.9,1], \varepsilon \in[0,1]$ and $d(t)=0.5 e^{-t}-1$. To illustrate the response of the closed-loop system, Figure 1 shows the results of a simulation with the system and design parameters set to $p_{i}^{*}=$ $1, \varepsilon=0.1, \quad c_{0}=-0.2, \quad k_{1}=0, \quad k_{2}=1$, $K=3, L_{1}=4, L_{2}=5$ and the initial conditions as $\left(x_{l}(0), y_{l}(0), \theta_{l}(0)+\varepsilon\right)=(1,1,-1), \widehat{z}(0)=$ $[0,-1,0]^{T}, P(0)=\operatorname{diag}\{0.1,0.2,0.3\}$,

Notably the non-vanishing external disturbance $d(t)=0.5 e^{-t}-1$ will make exiting control methods inapplicable to this mobile robot system even if all state variables are measurable. However, the aforementioned controller is effective with our combination of the ESO and dynamic observer techniques. The simulation results show the necessity of considering non-vanishing external disturbances in the control design as well as the effectiveness of the proposed method.
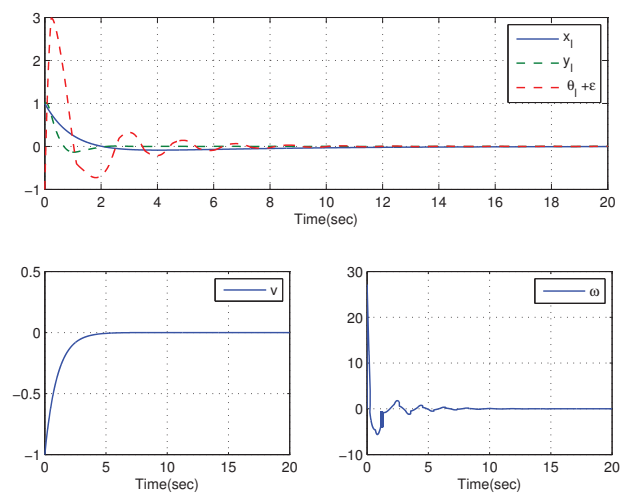

Figure 1 Responses of the closed-loop system.

\section{Acknowledges}

This work is supported by National Natural Science Foundation of China under Grants 61673243, U1713209 and Ministry of Education Key Laboratory of Measurement and Control of CSE under Grant MCCSE2017A0.

\section{References}

1 Jiang, Z.P. Robust exponential regulation of nonholonomic systems with uncertainties. Automatica, 2000, 36(2): 189-209.

2 Liu, Y.G. and Zhang, J.F. Output feedback adaptive stabilization control design for nonholonomic systems with strong nonlinear drifts. International Journal of Control, 2005, 78(7): 474-490.

3 Xi, Z.R., Feng, G., et al. Output feedback exponential stabilization of uncertain chained systems. Journal of the Franklin Institute, 2007, 344(1): 36-57.

4 Zheng, X.Y., Wu, Y.Q. Adaptive output feedback stabilization for nonholonomic systems with strong nonlinear drifts. Nonlinear Analysis: Theory, Methods \& Applications, 2009, 70(2): 904-920.

5 Han, J.Q. From PID to active disturbance rejection control. IEEE Transactions on Industrial Electronics, 2009, 56(3): 900-906.

6 Chen, W.H. Disturbance observer based control for nonlinear systems. IEEE/ASME Transactions on Mechatronics, 2004, 9(4):706-710

7 Liu R J, She J H, Wu M, et al. Robust disturbance rejection for a fractional-order system based on equivalent-input-disturbance approach. Sci China Inf Sci, 2018, 61(7):070222

8 Huang, J.S., Wen, C.Y., Wang,W., Jiang, Z.P. Adaptive output feedback tracking control of a nonholonomic mobile robot. Automatica, 2014, 50: 821-831.

9 Wu, Y.Q., Yu, J.B. and Zhao, Y. Further results on global asymptotic regulation control for a class of nonlinear systems with iISS inverse dynamics. IEEE Transactions on Automatic Control, 2011, 56(4): 941-946. 